\title{
1 The histone methyltransferase NSD3 contributes to cohesin loading during
} 2 mitotic exit

3

4

5

6

7

8

Grégory Eot-Houllier ${ }^{1,3 *}$, Laura Magnaghi-Jaulin ${ }^{1,2,3}$, Gaëlle Bourgine ${ }^{1}$, Erwan Watrin ${ }^{1}$ and Christian Jaulin ${ }^{1,2^{*}}$

${ }^{1}$ CNRS UMR 6290, University of Rennes1, IGDR (Institut de Génétique et Développement de Rennes), 35043, Rennes cedex, France.

${ }^{2}$ Present address: University of Rennes, EHESP, Inserm UMR 1085, Irset (Institut de Recherche en Santé, Environnement et Travail), 35000, Rennes, France.

${ }^{3}$ Equal contribution

*Corresponding authors

\section{Abstract} of the cohesin complex with chromatin. Phosphorylation / dephosphorylation and acetylation / deacetylation of histones and of cohesin components ensure correct establishment of cohesion during S phase and its proper dissolution during mitosis. In contrast, little is known about the contribution of methylation to the regulation of sister chromatid cohesion. We performed a RNA interference-mediated inactivation screen against 14 histone methyltransferases of the SET domain family that highlighted NSD3 as a factor essential for sister chromatid cohesion in mitosis. We established that NSD3 ensures proper level of the cohesin loader MAU2 and of cohesin itself onto chromatin at mitotic exit. Consistent with its implication in the loading of kollerin and cohesin complexes onto chromatin, we showed that NSD3 associates with chromatin in early anaphase prior to that of MAU2 and RAD21 and dissociates from chromatin upon cell's entry into prophase. Finally, we demonstrated that of the two NSD3 variant that exist in somatic cells, the long form that carries the methyltransferase activity is the one that acts in cohesion regulation. Taken together, these results describe a novel factor associated with histone methylation in cohesin loading. 


\section{Introduction}

To ensure faithful segregation of replicated DNA to daughter cells in mitosis, sister chromatids are held together during replication and their cohesion must persist at centromeres during the early stages of mitosis. Sister chromatid cohesion allows correct chromosome biorientation towards spindle poles and establishment of tension between kinetochores. In human somatic cells, sister chromatid cohesion is provided by a protein complex called cohesin composed of the three core subunits SMC1A, SMC3, and RAD21, which form a ring that can topologically embrace two chromatin fibres [1]. Cohesin core complex is further bound by regulatory subunits, the Scc3 homologs SA-1 or SA-2, PDS5A or PDS5B and WAPL [2, 3]. Cohesin is loaded onto chromatin during exit from mitosis by the NIPBL/MAU2 complex, also named Kollerin $[4,5]$. NIPBL contains the cohesin loading activity, whereas MAU2 facilitates NIPBL binding onto chromatin [6-9]. Establishment of sister chromatid cohesion occurs during DNA replication when sister chromatids are synthesised [1]. From this stage onward until early mitosis, Sororin binds to cohesin complex where it antagonizes WAPL anti-cohesive activity [10]. Upon mitotic entry in vertebrate cells, the mitotic kinases CDK1, PLK1 and Aurora B phosphorylate cohesin components and sororin, thereby rendering cohesin sensitive to WAPL activity, which leads to the dissociation of cohesin molecules from the chromosome arms, in a process known as the prophase pathway [11, 12]. During this process, sister chromatid cohesion is protected from WAPL action at the centromere by the protein Shugoshin 1 (SGO1) and the mitotic kinase Haspin. SGO1 competes with WAPL for cohesin ring association, and the SGO1 associated protein phosphatase 2A (PP2A) is believed to counterbalance the phosphorylation of cohesin and Sororin $[13,14]$. At the same time, Haspin binds to PDS5 and prevent interaction with WAPL $[15,16]$. Once all kinetochore are properly attached to microtubules at metaphase, the spindle assembly checkpoint is turned off, which leads to the activation of the endoprotease separase that cleaves cohesin subunit RAD21, thereby allowing opening of cohesin ring, its dissociation from centromeric regions and segregation of the two sets of chromosomes [17].

The maintenance of sister chromatid cohesion at centromere until the metaphase-toanaphase transition is linked to a combination of several histone phosphorylations. They form docking sites for effectors that counterbalance the cohesin dissociation induced by the prophase pathway [18-22]. Despite its presence at centromeres, a role of histone methylation in protecting centromere cohesion remains elusive [23]. Although Heterochromatin Protein 1 (HP1) favours Haspin recruitment, and possibly that of SGO1 also, at centromeres, whether HP1 association to $\mathrm{H} 3 \mathrm{~K} 9$ me3 nucleosome is required for centromeric cohesion is still an open question [24-26]. Furthermore, preventing di-methylation of H3K4 at centromere by artificial acetylation through HDAC3 depletion correlates with a premature separation of sister chromatids in early mitosis [27]. However, the associated molecular mechanism remains to be established. 
H3K4 methylation (mono-, di- and tri-methylation) is catalysed by protein macrocomplexes that belong to the SET domain methyltransferase family [28, 29]. These methyltransferases are categorised into subfamilies that include the Nuclear receptor binding SET Domain protein (NSD) subfamily, which comprises 3 members: NSD1, NSD2 (also known as WHSC1, for Wolf-Hirschhorn Syndrome candidate 1) and NSD3 (WHSC1L1 for WHSC1 protein Like 1). NSD methyltransferases act as oncoproteins in different types of cancers [30, 31] and are specific to H3K36 [32-36]. However, NSD3 contribution to H3K36 methylation seems to be less efficient as compared to that of NSD2. Moreover, a contribution to H3K4 di-methylation was shown following overexpression of the WHISTLE (WHSC1-like 1 isoform 9 with methyltransferase activity to lysine) isoform of NSD3 in NIH3T3 cells [32, $34,36,37]$. In addition to their SET domain, NSD family members are characterised by the presence of seven domains that bind to modified histones: five PHD domains (Plant Homeo Domain) that recognize specific DNA sequences together with histone PTMs (methylated Lysine or Arginine, acetylated Lysine), and two proline and tryptophan rich domains ( PWWP) which bind methylated lysines [37-39].

In somatic cells, NSD3 messenger RNA contains 24 exons and leads to the expression of two protein isoforms called NSD3L and NSD3s [37, 38, 40] while a third isoform, called WHISTLE, is specifically expressed in the testis from a downstream promoter [37]. The 1437 amino acid (aa) long form, NSD3L, includes the SET methyltransferase domain located in its carboxy-terminus while the 647 aa short form, NSD3s, results from an alternative splice and lacks the SET domain and, thus, the methyltransferase activity. NSD3s also differs from the long form in its last 620-647 aa with only one PWWP domains that bind di-methylated on lysine 36 (H3K36me2) [41]. The one PWWP domain of NSD3s is required for the function of BRD4NSD3s-CHD8 complex in sustaining leukemia state, suggesting a combinatorial pathway between H3K36me2 nucleosomes and acetylated nucleosomes bound by BRD4 to regulate gene expression at concerned super-enhancers regions.

In the present work, we report the identification of NSD3 as a SET family member whose inactivation resulted in precocious sister chromatid separation in early mitosis. We highlight the requirement of NSD3 for interphase cohesion in post-replicative cells and show that NSD3 is involved in cohesin loading at mitotic exit. We also describe that NSD3 loads onto chromatin in early anaphase prior to the association of the NIPBL/MAU2 cohesin loader complex with chromatin. Finally, we demonstrate that the role of NSD3 in cohesin loading is mediated by the NSD3L isoform that contains the SET methyltransferase domain. 


\section{Results}

\section{NSD3 contributes to the maintenance of sister chromatids cohesion in mitosis}

We have previously reported that depletion of Histone DeACetylase 3 (HDAC3) induces precocious sister chromatid separation during mitosis prior anaphase onset. This effect is accompanied by a forced acetylation of $\mathrm{H} 3 \mathrm{~K} 4$ and a loss of $\mathrm{H} 3 \mathrm{~K} 4 \mathrm{me} 2$ at centromeres, suggesting a contribution of centromeric H3K4me2 to sister chromatid cohesion in human. Given that mutated histone versions cannot be specifically targeted to centromere and, further, that H3K4 PTMs contribute to several chromatin-based processes beside centromere -in particular gene regulation- pleiotropic effects resulting from the expression of a histone $\mathrm{H} 3$ version that can no longer be modified at the K4 residue are expected [42]. As an alternative, we performed an RNAi-mediated inactivation screen for defective mitotic cohesion focussing on 14 human SET domain-containing methyltransferases. In that aim, three different siRNAs per SET methyltransferase were transfected in Kyoto HeLa cells, and mitotic chromosome spreads were prepared and analysed for mitotic cohesion defects (Figure 1A). As shown in Supplementary Figure 1, a single methyltransferase, NSD3, could be identified as leading to a significantly increased proportion of prometaphase cells displaying separated sister chromatids (Figure 1B-C, Figure S1). This screen therefore revealed NSD3 as a methyltransferase important for sister chromatid cohesion in mitosis.

As NSD3 is involved in gene expression regulation, it remains possible that the cohesion defects we observed could arise from the altered expression of cohesin components. To test this possibility, we analysed total amounts of cohesin subunits and of cohesin loader MAU2 by immunoblotting experiments of synchronised, RNAi treated cells. As shown in Supplementary Figure 2, western blot analyses revealed that the expression levels of RAD21, SA1/SA2, SMC3 and of MAU2 were not altered following NSD3 depletion neither in nocodazole arrested-cells nor after block release. Moreover, these immunoblotting experiments also indicated that global di-methylation levels of H3K4 and H3K36 remained unaffected by NSD3 inactivation (Figure S2), indicating that NSD3 involvement in sister chromatid cohesion is independent both of the global disruption of $\mathrm{H} 3 \mathrm{~K} 4$ and $\mathrm{H} 3 \mathrm{~K} 36$ di-methylation status and of altered expression of cohesin components and of MAU2.

\section{NSD3 is involved in cohesin loading regulation}

An increase in the proportion of mitotic cells exhibiting separated sister chromatids, such as the one we observed upon NSD3 inactivation, can arise either from the accumulation of prometaphase cells with defective mitotic cohesion or from a premature entry of cells into anaphase. In order to discriminate between these two possibilities, we first investigated the status of the spindle assembly checkpoint activity by analysing both the presence of the mitotic 
checkpoint complex kinase BUB1 and of the centromeric cohesin protector SGO1 at pericentromeric regions by indirect immunofluorescence experiments. As shown in Figure 2, BUB1 kinase could be detected at kinetochores of cells displaying defective cohesion, indicating that the spindle checkpoint in still active and that, therefore, cells are arrested in prometaphase (Figure 2A). Similarly, SGO1 could be detected at centromeres of separated sister chromatids following NSD3 depletion, confirming that these cells were indeed blocked in prometaphase (Figure 2B) instead of having entered anaphase. Altogether these observations indicate that NSD3 is required for a proper sister chromatid cohesion in mitosis and suggests that this role does not depend on SGO1-mediated protection of centromeric cohesin.

Thus, we next aimed at determining whether defective cohesion detected in mitotic cells after NSD3 depletion was already present in interphase post-replicative cells. In that goal, we analysed cohesion between sister chromatids in interphase cells by DNA Fluorescent In Situ Hybridization (FISH) using probes that bind to chromosome 11 centromere regions in cells synchronised in G2-phase (Figure 2C-E). Cohesion status was assessed by measuring distances between paired FISH signals in control cells and in cells depleted for NSD3 as well as cells depleted for RAD21 used as a positive control of defective cohesin. In control cells, mean distance between paired dots was $0.52 \mu \mathrm{m}$ and increased to $0.68 \mu \mathrm{m}$ in RAD21-depleted cells and to $0.67 \mu \mathrm{m}$ upon NSD3 depletion. These experiments revealed that NSD3 depletion resulted in defective sister chromatid cohesion in G2 cells, indicating that NSD3 contributes to cohesion before cells enter mitosis, i.e. in interphase.

Then, we asked whether NSD3 could be involved in the loading of cohesin and/or of kollerin onto chromatin, a process that takes place at mitotic exit. In that aim, control and NSD3depleted cells were synchronised by single thymidine arrest-and-release coupled to mitotic arrest using the mitotic spindle poison nocodazole (Figure 3A). Cells were harvested at different time points after release from the mitotic arrest and fractionated into soluble and chromatin fractions that were analysed by immunoblotting experiments after SDS-PAGE. As shown in Figure 3B, NSD3 inactivation did not impact on cell's mitotic exit as both condensin II complex subunit HCAP-D2 unloading and histone H3 Serine10 de-phosphorylation occurred with identical kinetics between control and NSD3 inactivated cells. However, accumulation of cohesin subunits on chromatin over time was reduced by around $50 \%$ in NSD3-depleted cells when compared to that in control cells. Remarkably, similar albeit more modest $30 \%$ reduction was also observed for the kollerin subunit MAU2. This reduction in detected protein signals was not due to reduced amounts of chromatin as both chromatin associated enzyme topoisomerase II and histones were present at similar levels in control and NSD3-depleted cells.

This experiment showed that NSD3 inactivation leads to reduced amounts of cohesin and MAU2 loaded onto chromatin during exit from mitosis, indicating that NSD3 contributes to the loading of both cohesin and kollerin complexes at the end of mitosis. 


\section{NSD3 is released from chromatin at mitosis onset and reloaded in early anaphase}

As shown in Figure 3, immunoblotting analysis of chromatin fractions revealed that both NSD3 long and short variants were absent from chromatin in mitosis-arrested control cells and progressively accumulated upon release and progression to G1 phase.

To characterise this dynamic behaviour in more details, we aimed at analysing the localization of NSD3 at the different stages of the cell cycle by indirect immunofluorescence experiments. First, NSD3 antibody staining specificity was determined by RNAi-mediated inactivation of NSD3 followed by antibody staining that resulted in a strong reduction of the NSD3 antibody fluorescence signal down to background signal (Supplementary Figure 3).

Next, we performed NSD3 antibody staining in an asynchronous HeLa cell population and monitored corresponding signals in interphase cells and in mitotic cells using both chromosome morphology and H3S10 phosphorylation mark staining as a mean to identify the different stages of mitosis. These immunostaining experiments were performed with and without detergent-based pre-extraction of the soluble pool of proteins (Figure 4A-B). Fluorescence microscopy imaging of these samples revealed that NSD3 signal is associated with chromatin in interphase and prophase cells and is essentially absent from chromosomes from early prometaphase until metaphase (Figure 4B). A very weak NSD3 signal could still be detected on chromatin in prometaphase cells after pre-extraction. To test if that remaining signal is due to the presence of a fraction of NSD3 on chromatin, we looked for the presence of NSD3 on chromosome spreads. As shown in Figure 4C, the weak labelling observed in control siRNA treated prometaphase cells did not co-localize neither with whole chromosomes nor with centromere regions as revealed by CREST serum co-staining. No obvious difference was observed when mock-siRNA treated prometaphase cells were compared to NSD3-siRNA treated prometaphase cells (Figure 4C) indicating that the weak fluorescence signal observed on pre-extracted prometaphase cells (Figure 4B) represents non-specific background. After the metaphase-to-anaphase transition, NSD3 signal is detected on chromatin as soon as in early anaphase (Figure 4B). These results were confirmed using two doxycycline-inducible cell lines we established and that allow specific expression of the long or the short isoforms of NSD3 fused to a LAP (Localization and affinity purification) tag containing in particular an Emerald GFP (Figure S4 and next section), altogether indicating that NSD3 isoforms both associate with chromatin in interphase and early prophase, dissociate from chromosomes in prophase and reassociate with chromosome at anaphase, similar to the dynamic behaviour of cohesin and kollerin complexes.

Finally, we repeated these fluorescence microscopy experiments in the two LAP-NSD3 cell lines to assessed differential chromatin association/dissociation of NSD3 relative to that of cohesin and of kollerin (Figure 4D-E, Figure S5). These experiments showed that association with chromatin of LAP-NSD3L occurs prior to that of RAD21 and of MAU2 (Figure 4D-E, 
Figure S5) and in an identical fashion for LAP-NSD3s (not shown). Thus, NSD3 is removed

\section{Sister chromatid cohesion depends on the long form of NSD3}

Our work has revealed that RNAi-mediated inactivation of NSD3 resulted in cohesin and kollerin loading and in subsequent cohesion defects both in interphase and in mitotic cells. As the siRNA we used targeted both long and short isoforms of NSD3 protein, we aimed at determining whether one or both isoforms were involved in cohesion regulation. In that goal, we designed isoform-specific siRNAs (two per isoforms) that selectively depleted each of them in an efficient manner as revealed by immunoblotting experiments (Figure 5A). We then compared the ability of these isoform-specific siRNAs to induce precocious sister cohesion defects in mitotic cells as compared to that of siRNAs targeting both of them. As shown in Figure 5B, both siRNAs targeting the long form of NDS3 resulted in defective mitotic cohesion, while the siRNAs targeting the short form did not have any effect on cohesion despite an efficient protein amount reduction. This result indicates that the long form of NSD3, and not its short version, is involved in sister chromatid cohesion.

To confirm this result, we made use of the cell line we established (see above and Figure 5C-D) that expresses a LAP-tagged version of NSD3L that has been rendered resistant to NSD3 RNAi (see Methods for details) and we assessed its ability to rescue defective mitotic cohesion upon depletion of NSD3L\&s and NSD3L using specific siRNAs. Treated cells were analysed by scoring the percentage of prometaphase cells exhibiting precocious sister chromatid separation in each condition with and without doxycycline induction (Figure 5D-E). The cell line heterogeneity in expressing LAP-NSD3L allowed us to use GFP-negative cells as an internal control (Figure 5C). In the absence of doxycycline, we confirmed that depleting either both NSD3 isoforms or NSD3L only resulted in premature sister chromatid separation, identical to the situation in doxycycline-treated cells that were negative for GFP signals (Figure 5E, black and grey bars, respectively). By contrast, in cells where LAP-NSD3L was expressed (GFP positive cells), the proportion of mitosis displaying precocious sister chromatid separation was significantly reduced (Figure 5E, green bars), which indicated that ectopic correction of NSD3L protein amount was able to rescue defective mitotic cohesion, thereby establishing NSD3L as the isoform required for proper sister chromatid cohesion.

\section{Discussion}

Sister chromatid cohesion is established during $\mathrm{S}$ phase, depends on cohesin which loading onto chromatin requires the kollerin complex and stands essential for proper 
chromosome segregation at anaphase. Here, we show that NSD3 is required for the recruitment of the cohesin loader MAU2 and, consequently, to that of cohesin to chromatin at mitosis exit. Therefore, we propose that precocious sister chromatid separation observed in mitotic cells upon NSD3 inactivation is an indirect consequence of defective kollerin recruitment and, as a consequence, of cohesin loading onto chromatin. In full agreement with its function in promoting Kollerin targeting on chromatin, our observation showed that the dynamic localisation behaviour of NSD3 is similar to that of MAU2 and of cohesin [5]. We also noticed that NSD3 binds to chromatin during anaphase shortly before MAU2 and RAD21. Therefore, NSD3 is unlikely to interact with NIPBL/MAU2 prior to their targeting to the chromatin. Rather, NSD3 may promote kollerin loading onto chromatin by facilitating its binding at the end of mitosis. Consistent with this possibility, yeast MAU2 homologue Scc4 is necessary to load Scc2 onto chromatin in vivo, but has no affinity to bind DNA, implying the requirement for a protein receptor [6-8]. NSD3 represents a good candidate to play such a role as receptor, thereby triggering the recruitment of NIPBL/MAU2 complex via direct protein-protein interaction. Indeed, recent reports have shown that NSD3 interacts with the transcriptional regulator BRD4, which in turn interacts with NIPBL through its extra-terminal domain and stabilizes it on specific chromatin regions in vertebrate cells [43, 44]. It is thus tempting to propose that BRD4/NSD3 acts as a platform that binds and loads NIPBL/MAU2 onto chromatin.

Alternatively, the contribution of NSD3 could be to render chromatin regions amenable for Kollerin loading via chromatin remodelling. Consistent with this view, it has been reported that NSD3 acts as an adaptor protein bridging the BET domain of BRD4 and the chromatin remodelling factor CHD8. Moreover, two reports from Franck Uhlmann's laboratory have demonstrated that the chromatin remodeler RSC (Remodels the Structure of Chromatin) acts as a chromatin receptor by interacting physically with the Scc2/Sc4 complex in the yeast S.pombe [45-47]. They have also shown that RSC plays also a conserved role by promoting nucleosome removal in order to render the DNA naked for the binding of the cohesin complex independently of Scc2/Scc4 [46, 47]. Thus, one can imagine that similarly to the yeast process, BRD4/NSD3/CHD8 complex acts in human kollerin recruitment through two complementary mechanisms. Firstly, CHD8 nucleosome sliding activity displace nucleosomes to create a nucleosome free region. Secondly, BRD4 ensures the physical interaction with the Kollerin complex [48]. In this context, as proposed by Shen \& al [44], NSD3 bridge BRD4 and CHD8 and thus would generate an integrated molecular process of kollerin recruitment onto chromatin. Similarly, additional chromatin remodellers have been described to contribute to cohesin loading $[49,50]$. Whether NSD3 requirement for Kollerin loading onto chromatin involves chromatin remodelling will require dedicated investigations.

In addition, our results revealed that MAU2 and cohesin loading onto chromatin involves only the long form of NSD3. Domains interacting with BRD4 and CHD8 are common for both 
NSD3 isoforms [44]. Therefore, if physical interaction with these two partners would be sufficient for the NSD3 regulation of cohesin loading, one could have expected that only depletion of both isoforms would prevent cohesin loading. In contrast to this possibility, we showed that only the depletion of NSD3L and not that of NSD3s, resulted in defective cohesion. This implies that, in addition to its possible association with BRD4 and CHD8, NSD3L function in kollerin loading requires further properties, such as additional co-factors or enzymatic activity. In accordance with the latter hypothesis, NSD3L is the one NSD3 isoform that contains the methyltransferase activity. It is thus tempting to propose that NSD3L acts by generating post-translational modifications of yet unidentified substrates. In our current state of knowledge, H3K36me2 represents the best candidate for further investigations concerning the contribution of NSD3 to the recruitment of cohesin loaders and cohesin onto chromatin. Indeed, H3K36me2 is found in active gene promoters and NIPBL was also enriched to those region in human cells [51-53]. One can speculate that NSD3L would act to render chromatin prone for further kollerin targeting by methylating histones. The use of a mark that is stable throughout mitosis, as $\mathrm{H} 3 \mathrm{~K} 36 \mathrm{me} 2$, could be essential for the epigenetic inheritance of cohesin loading sites.

\section{Materials and methods}

\section{Antibodies.}

Antibodies dilutions for western blotting (WB) and immunofluorescence (IF) are indicated below. The antibodies used were: rabbit anti-NSD3 (Proteintech 11345-1-AP) WB: 1/1000, IF: 1/1000 ; mouse anti-RAD21 (Millipore ref 05-908) WB:1/5000, IF: 1/1000 ; rabbit anti-MAU2 (Abcam Ab183033) WB: 1/5000, IF: 1/1000 ; mouse anti-SA2 (Santa Cruz sc-81852) WB:1/500 ; rabbit anti-SMC3 WB: 1/1000 [54], rabbit anti-hCAP-D2 1/1000 [55], mouse antiTopoisomerase II $\alpha(\mathrm{Ki}-\mathrm{S} 1)$ Millipore (MAB4197) WB: 1/2000 ; mouse anti-p-H3S10 (Millipore 05-806) WB: 1/10 000, IF: 1/20 000, rabbit anti-H3K4me2 (C64G9) (Cell Signaling Technology \#9725) WB: 1/5000 ; rabbit anti-H3K36me2 (Active Motif 39056) WB: 1/5000 ; mouse anti-H4 (Abcam Ab31830) WB: 1/2000 ; rabbit polyclonal anti-actin (Sigma A5060) WB: $1 / 10$ 000; mouse anti- $\alpha$-tubulin (Sigma T5168) WB: 1/5000 ; mouse monoclonal antiBub-1 (Sigma B0561) IF: 1/100 ; mouse anti-GFP (Roche 11814460001) WB: 1/500, IF: 1/500. Rabbit anti-Sgo1 antibody was a gift from Prof. Yoshinori Watanabe (Japan) IF: 1/1000 [56]; Human CREST serum was a gift from Dr Isabelle Bahon-Riedinger (France) IF: 1/4000. Horseradish peroxidase coupled secondary antibodies (Jackson Immunoresearch) (WB: Mouse 1/5000 Rabbit 1/25000) and Alexa Fluor-coupled secondary antibodies from Invitrogen (IF: 1/1000) were used for WB and IF detection, respectively.

\section{Plasmid construction and cell line generation.}


A fragment containing an inducible TRE tight promoter and a LAP tag (Flag tag followed by emerald Green Fluorescent Protein tag) was inserted at the XhoI site of the pBSKDB-CAGrtTA2sM2-IRES-tSkid-IRES-Neo plasmid (Addgene \#62346), generating a vector named pGEH_ind_LAP-C. In this vector, NSD3s or NSD3L were cloned in frame in C-terminal of the LAP tag after PCR amplification from plasmids pMSCV_MigR1_NSD3short and pMSCV_MigR1_NSD3long, kindly given by CR.Vakoc [44]. All PCR were realized with the Q5 high fidelity polymerase (NEB M0493S) and all constructions and intermediates were generated by NEBuilder HiFi DNA assembly cloning kit according to manufacturer recommendations (NEB E5520S). Plasmids and their maps will be provided upon request.

For generation of HeLa-LAP-NSD3L and HeLa-LAP-NSD3s cell lines, $5 \mu \mathrm{g}$ of corresponding plasmids, supplemented with $10 \mu \mathrm{l}$ of P3000 in $125 \mu$ l Opti-MEM (ThermoFischer Scientific 31985062), were mixed with $7.5 \mu$ l lipofectamine 3000 in $125 \mu$ l Opti-MEM. After 5 min incubation at room temperature, mix containing plasmids were transfected in HeLa-Kyoto seeded the day before in $2.25 \mathrm{ml}$ of complete medium in 6 well plates. $24 \mathrm{~h}$ later, cells were trypsinized and 1/10 or 1/50 of the cells was seeded in a $150 \mathrm{~mm}$ culture dish in $25 \mathrm{ml}$ of complete medium containing $1 \mathrm{mg} / \mathrm{ml}$ of G418 (ThermoFischer Scientific 11811023). Selection lasted for 2-3 weeks with replacement of the antibiotics supplemented medium each 2-3 days. Then, cells were induced for LAP-NSD3 expression with $2 \mu \mathrm{g} / \mathrm{ml}$ of doxycyline (Merck D9891$1 \mathrm{G}$ ) for $48 \mathrm{~h}$ and GFP positive cells were FACS sorted using the CytomeTRI platform (Biosit SFR UMS CNRS 3480 - INSERM 018 - Rennes) and cultured for another 2 weeks under selection pressure, but without induction. Then, cells lines were subcloned by limiting dilution in 96 well plate and after 2 weeks of selection, around 30 clones were tested for LAP-NSD3 expression for each NSD3 variant. Despite all this process, no homogeneous clonal cell line could be obtained. For the LAP-NSD3s construct, we always obtained clones that expressed the fused protein constitutively. By contrast, expression of LAP-NSD3L in the selected cell lines was inducible as expected.

\section{Cell culture and treatment}

All experiments presented in this paper were performed with HeLa-Kyoto cells or cell lines constructed from these cells. All cell lines were cultured in Dulbecco's Modified Eagle's Medium with glutamine analogue Glutamax (Thermofischer Scientific 31966047), supplemented with $10 \%$ fetal bovine serum and a cocktail of penicillin/streptomycin antibiotics (Thermoficher Scientific 15140-122) at $100 \mathrm{U} / \mathrm{ml}$ and $100 \mu \mathrm{g} / \mathrm{ml}$ final concentration, respectively. Stable cell lines were maintained in culture with G418 $(1 \mathrm{mg} / \mathrm{ml})$. Doxycycline was used at $1 \mu \mathrm{g} / \mathrm{ml}$ in medium to induce expression of the exogenous tagged protein and was replaced with fresh doxycyline-containing medium after $48 \mathrm{~h}$ when it was necessary. To synchronize cells, thymidine (Merck T1895-1G) was added to the medium 8 hours post- 
transfection at a final concentration of $2 \mathrm{mM}$ and cells were cultured for $24 \mathrm{~h}$, followed by two successive 3 min PBS washes and incubation with complete medium supplemented with 100 $\mathrm{ng} / \mathrm{ml}$ nocodazole (Merck M1404) for another 16h to arrest cells in prometaphase. Then, cells were harvested by mitotic shake-off, washed as described for the previous release and seeded in fresh complete medium in new plates. All medium were pre-warmed at $37^{\circ} \mathrm{C}$ before using in synchronisation experiments.

\section{siRNA transfection}

HeLa cells were transfected with $20 \mathrm{nM}$ siRNA for $72 \mathrm{~h}$, unless otherwise stated in the figure legends, with Hiperfect (Qiagen) following manufacturer recommendations. As a general guideline, for a 6 well transfection, $10 \mu \mathrm{l}$ of Hiperfect reagent and $2,5 \mu 1$ of $20 \mu \mathrm{M}$ stock siRNA were mixed in 87,5 $\mu 1$ of Opti-MEM and added to 300000 HeLa cells seeded in suspension in $2,4 \mathrm{ml}$ of complete medium. Medium was replaced after $48 \mathrm{~h}$ transfection and cells were trypsinized and diluted if necessary. siRNA used in the experiments were described below and were purchased from Qiagen of Dharmacon. Those used for the methyltransferase screening can be communicated upon request.

\begin{tabular}{|l|l|l|l|}
\hline $\begin{array}{l}\text { siRNA } \\
\text { name }\end{array}$ & Gene targeted & Sequence sense & Sequence antisense \\
\hline NSD3-a & NSD3 & CGAGAGUAUAAAGGUCAUAdTdT & UAUGACCUUUAUACUCUCGdTdA \\
\hline NSD3-b & NSD3 & GACCAAGAUCUGUGCUGAAdTdT & UUCAGCACAGAUCUUGGUCdGdT \\
\hline NSD3-c & NSD3 & AAUGGGUAUCCAUCAUCAAdTdT & UUGAUGAUGGAUACCCAUUdTdG \\
\hline NSD3L-A & NSD3L-3'UTR & GGUCUUAAUUGGAGAGAAUdTdT & AUUCUCUCCAAUUAAGACCdAdG \\
\hline NSD3L-B & NSD3L-3'UTR & GGGACAGGCUAUUGGACAAdTdT & UUGUCCAAUAGCCUGUCCCdAdT \\
\hline NSD3s-a & NSD3s-3'UTR & GGUUUGAGCUGGAUGGGUAdTdT & UACCCAUCCAGCUCAAACCdGdA \\
\hline NSD3s-b & NSD3s-3'UTR & UCAGAAACUCAUCGGAAAUdTdT & AUUUCCGAUGAGUUUCUGAdAdA \\
\hline Luc & Luciferase & CGUACGCGGAAUACUUCGAdTdT & UCGAAGUAUUCCGCGUACGdTdT \\
\hline Rad21 & Rad21 & CGAUGAGCCCAUUAUUGAAdTdT & UUCAAUAAUGGGCUCAUCGdTdT \\
\hline
\end{tabular}

\section{Chromosome spreads}

Cells harvested from a 6 well plate were subjected to hypotonic shock in $4.5 \mathrm{ml}$ of $75 \mathrm{mM} \mathrm{KCl}$ for $15 \mathrm{~min}$ at room temperature and $500 \mu \mathrm{l}$ of Carnoy's fixative ( 3 vol methanol/1 vol acetic acid) was added. Cells were centrifuged at $250 \mathrm{~g}$ and the pellet was resuspended in $5 \mathrm{ml}$ of Carnoy's fixative. This operation was repeated 3 mores times and the pellet were keep at $-20^{\circ} \mathrm{C}$ in $300 \mu 1$ of Carnoy's fixative overnight. Then, we dropped $30 \mu 1$ of cells on a dry slide, let them dry for $2 \mathrm{~h}$ at room temperature, and incubate slides for $5 \mathrm{~min}$ in fresh 5\% GIEMSA (Merck 1.09203) solution diluted in $100 \mathrm{ml}$ Gurr buffer (ThermoFisher scientific 10582-013). 
Following 3-5 washes in distilled water, giemsa stained cells were mounted with entellan. Alternatively, for immunofluorescence on chromosome spreads, following hypotonic shock around 50000 swollen cells in $200 \mu 1$ of $\mathrm{KCl} 75 \mathrm{mM}$ were cytospun on a slide for 5 minutes at 900 rpm (Cytospin 4; Thermo Scientific) and were fixed in 3\% paraformaldehyde/PBS for further immuno-labelling.

\section{Immunofluorescence}

When stated, soluble contents of cells were pre-extracted by incubation for $1 \mathrm{~min}$ in $0,1 \%$ Triton (Merck T8787-50ML) diluted in PBS 1X (ThermoFisher Scientific 10010-015). Otherwise, cells were fixed 10 min in 4\% Paraformaldehyde (EMS 15710) diluted in PBS 1X pH 7.2pH7.4 final. Slides or coverslips were then washed three times 5 min in PBS 1X, permeabilized with $0.1 \%$ Triton X-100 for 10 min, washed three times in PBS $1 \mathrm{X}$ and blocked by incubation with 5\% FCS in PBS $1 \mathrm{X}$ for $1 \mathrm{~h}$ min at room temperature. This last solution was used to dilute primary and secondary antibodies. Slides or coverslips were then incubated overnight at $4{ }^{\circ} \mathrm{C}$ with primary antibodies, washed three times with PBS $1 \mathrm{X}$ and then incubated for 1 hour at room temperature with fluorochrome-conjugated secondary antibodies. The DNA was stained with DAPI (100 ng/ml in PBS) and slides were mounted in Prolong-Gold medium (ThermoFisher Scientific P36982).

\section{Microscopy and image analysis}

Images were acquired with an epifluorescence microscope (Zeiss AxioImager.M2) equipped with Zeiss "Plan-Apochromat" 40x/1.3 and 63x/1,40 oil objectives, a Coolsnap HQ ${ }^{2}$ CCD camera (Photometrics) and Zeiss Axiovision software (version 4.2). Signals were quantified with ImageJ software (Rasband, W.S., ImageJ, US National Institutes of Health, Bethesda, Maryland, USA, http://rsb.info.nih.gov/ij/, 1997-2005).

\section{Fluorescent In Situ Hybridization}

DNA FISH was performed as previously described [57], with the exception that cells were fixed with Carnoy's fixative as described above and processed for FISH after spreading on glass slides. Only pairs for which the dots could be clearly resolved were considered in the analysis. The probe used for FISH targets the alpha-satellite sequence AgGgTtTcAgAgCtGcTc that is specific of the centromeric region of the 11 chromosome. In the probe sequence, uppercases correspond to DNA whereas lowercases correspond to a locked nucleic acid, which is a modified RNA nucleotide in which the ribose moiety is modified with an extra bridge connecting the 2' oxygen and 4' carbon. The probe was coupled with an Alexa-488 fluorophore. 


\section{Cell extracts and western blotting}

427

428

429

430

431

432

433

434

435

436

437

438

439

440

441

442

443

444

445

446

447

448

449

450

451

452

453

454

455

456

457

458

459

460

461

462

For whole cell extracts, proteins were extracted by directly resuspending cells pellet in Laemmli buffer (60 mM Tris-HCl pH6.8, 10\% glycerol, 2\% SDS, $0.05 \%$ bromophenol blue, 5\% $\beta$ mercaptoethanol). When fractionation was required, cells were collected by trypsinization and washed once with ice-cold PBS. The final cell pellet was resuspended in extraction buffer (20 mMTris $\mathrm{pH}$ 7.5, $100 \mathrm{mM}$ sodium chloride, $5 \mathrm{mM}$ magnesium chloride, $0.2 \%$ NP-40, 10\% glycerol, $0.5 \mathrm{mM}$ dithiothreitol) supplemented with EDTA-free tablets of protease inhibitors (Merck 5892953001) and home-made phosphatase inhibitors cocktail (5 mM sodium fluoride, $10 \mathrm{mM} \beta$-glycerophosphate, $1 \mathrm{mM}$ sodium pyrophosphate and $0.2 \mathrm{mM}$ sodium orthovanadate final concentration respectively). Cells were lysed on ice by ten passages through a 27 -gauge needle. Lysates were incubated for 10 minutes on ice and were then centrifuged (12 $000 \mathrm{~g}$, 5 minutes, $4^{\circ} \mathrm{C}$ ) for collection of the soluble protein extract. The chromatin-containing pellet was washed four times with extraction buffer $\left(12,000 \mathrm{~g}, 5\right.$ minutes, $\left.4^{\circ} \mathrm{C}\right)$ and then directly resuspended in Laemmli buffer.

For immunoblotting, lysed cells were heated for 5 minutes at $95^{\circ} \mathrm{C}$. Samples were then subjected to SDS-PAGE in a 4-20\% polyacrylamide gradient gel (Biorad \#4561094) and transferred to ready-to-use PVDF membranes (Biorad \#1704156) with a Trans-Blot Turbo transfer system (Biorad). Following saturation for $1 \mathrm{~h}$ hour in PBS $1 \mathrm{X}$ containing 5\% milk and $0.1 \%$ tween-20, membranes were incubated overnight at $4{ }^{\circ} \mathrm{C}$ with primary antibodies and then for 1 hour at room temperature with horseradish peroxidase (HRP)-conjugated secondary antibodies, according to standard procedures. For NSD3 western blotting, saturation and incubation were performed with similar procedures, except that saturation and incubation buffer was supplemented with $10 \%$ milk instead of $5 \%$ and $150 \mathrm{mM} \mathrm{NaCl}$. For revelation, membranes were treated with the substrate HRP Immobilon ECL kit (Merck WBKLS0500) and signal was detected with an Amersham Imager 680 (GE Healthcare) or with Amersham hyperfilm ECL (Merck GE28-9068-35). Quantification of band signal intensity were realized with the Analyze/Gels tools of ImageJ.

\section{Statistics}

All statistical analysis were performed with GraphPad Prism v6.05 (GraphPad Software). For the comparison of PSCS or mitotic index between NSD3 depleted cell lines, we performed oneway analysis of variance (ANOVA) followed by Dunett post hoc analysis, assuming normal law for the repartition of the means. Comparisons of fluorescence intensity from a representative experiment were analysed in nonparametric Kruskall Wallis test followed by Dunn's multiple comparison correction. For the comparison of distance in FISH experiment, one-way ANOVA was used followed by Bonferroni's multiple comparisons test. For all statistical tests, an alpha risk of 0.05 was used. 


\section{Author contributions}

G.E.-H and L.M.-J designed and performed the experiments. G.B contributed to plasmid constructions. G.E-H, L.M-J and E.W analysed data. G.E-H and E.W wrote the manuscript. C.J. designed and supervised the project, provided funding and revised the manuscript.

\section{Competing interests}

The authors have no conflict of interest to declare.

\section{Acknowledgments}

We would like to especially thank Dr. Rozenn Gallais for her singular contribution to this study. We would like to thank Prof. Christopher Vakoc (Cold Spring Harbor Laboratory, USA), Dr. Christophe Escudé (National Museum of natural history, France), Prof. Yoshinori Watanabe (University of Tokyo, Japan), Prof. and Dr Isabelle Bahon-Riedinger (Rennes University Hospital, France) for providing reagents. We also thank Laurent Deleurme from the Flowcytometry platform CytomeTRI of the BIOSIT SFR UMS CNRS 3480 - INSERM 018 unit, for the FACS cell sorting of LAP-NSD3 HeLa cells lines. G.E.-H, L.M.-J, G.B and E.W are investigators at the CNRS. C.J. is investigator at INSERM. This work was funded by the French National Research Agency (ANR, project "EpiCentr"), the Région Bretagne (SAD grant), the Cancéropôle Grand Ouest, the Ligue Contre le Cancer (Comité Grand Ouest) and the Fondation ARC pour la Recherche sur le Cancer.

\section{References}

1. Nasmyth, K., and Haering, C.H. (2009). Cohesin: its roles and mechanisms. Annu Rev Genet 43, 525-558.

2. Makrantoni, V., and Marston, A.L. (2018). Cohesin and chromosome segregation. Curr Biol 28, R688-R693.

3. Nishiyama, T. (2019). Cohesion and cohesin-dependent chromatin organization. Curr Opin Cell Biol 58, 8-14.

4. Ciosk, R., Shirayama, M., Shevchenko, A., Tanaka, T., Toth, A., Shevchenko, A., and Nasmyth, K. (2000). Cohesin's binding to chromosomes depends on a separate complex consisting of Scc2 and Scc4 proteins. Mol Cell 5, 243-254.

5. Watrin, E., Schleiffer, A., Tanaka, K., Eisenhaber, F., Nasmyth, K., and Peters, J.M. (2006). Human Scc4 is required for cohesin binding to chromatin, sister-chromatid cohesion, and mitotic progression. Curr Biol 16, 863-874.

6. Chao, W.C., Murayama, Y., Muñoz, S., Costa, A., Uhlmann, F., and Singleton, M.R. (2015). Structural Studies Reveal the Functional Modularity of the Scc2-Scc4 Cohesin Loader. Cell Rep 12, 719-725.

7. Hinshaw, S.M., Makrantoni, V., Kerr, A., Marston, A.L., and Harrison, S.C. (2015). Structural evidence for Scc4-dependent localization of cohesin loading. Elife 4, e06057.

8. Murayama, Y., and UhImann, F. (2014). Biochemical reconstitution of topological DNA binding by the cohesin ring. Nature 505, 367-371.

9. Parenti, I., Diab, F., Gil, S.R., Mulugeta, E., Casa, V., Berutti, R., Brouwer, R.W.W., Dupé, V., Eckhold, J., Graf, E., et al. (2020). MAU2 and NIPBL Variants Impair the Heterodimerization of the Cohesin Loader Subunits and Cause Cornelia de Lange Syndrome. Cell Rep 31, 107647. 
10. Nishiyama, T., Ladurner, R., Schmitz, J., Kreidl, E., Schleiffer, A., Bhaskara, V., Bando, M., Shirahige, K., Hyman, A.A., Mechtler, K., et al. (2010). Sororin mediates sister chromatid cohesion by antagonizing Wapl. Cell 143, 737-749.

11. Nishiyama, T., Sykora, M.M., Huis In 't Veld, P.J., Mechtler, K., and Peters, J.M. (2013). Aurora $B$ and Cdk1 mediate Wapl activation and release of acetylated cohesin from chromosomes by phosphorylating Sororin. Proc Natl Acad Sci U S A 110, 13404-13409.

12. Sumara, I., Vorlaufer, E., Stukenberg, P.T., Kelm, O., Redemann, N., Nigg, E.A., and Peters, J.M. (2002). The dissociation of cohesin from chromosomes in prophase is regulated by Pololike kinase. Mol Cell 9, 515-525.

13. Kitajima, T.S., Sakuno, T., Ishiguro, K.I., lemura, S.I., Natsume, T., Kawashima, S.A., and Watanabe, Y. (2006). Shugoshin collaborates with protein phosphatase $2 A$ to protect cohesin. Nature.

14. Liu, H., Rankin, S., and Yu, H. (2013). Phosphorylation-enabled binding of SGO1-PP2A to cohesin protects sororin and centromeric cohesion during mitosis. Nat Cell Biol 15, 40-49.

15. Liang, C., Chen, Q., Yi, Q., Zhang, M., Yan, H., Zhang, B., Zhou, L., Zhang, Z., Qi, F., Ye, S., et al. (2018). A kinase-dependent role for Haspin in antagonizing Wapl and protecting mitotic centromere cohesion. EMBO Rep 19, 43-56.

16. Zhou, L., Liang, C., Chen, Q., Zhang, Z., Zhang, B., Yan, H., Qi, F., Zhang, M., Yi, Q., Guan, Y., et al. (2017). The N-Terminal Non-Kinase-Domain-Mediated Binding of Haspin to Pds5B Protects Centromeric Cohesion in Mitosis. Curr Biol 27, 992-1004.

17. London, N., and Biggins, S. (2014). Signalling dynamics in the spindle checkpoint response. Nat Rev Mol Cell Biol 15, 736-748.

18. Dai, J., Sullivan, B.A., and Higgins, J.M. (2006). Regulation of mitotic chromosome cohesion by haspin and aurora B. Dev Cell 11, 741-750.

19. Hengeveld, R.C.C., Vromans, M.J.M., Vleugel, M., Hadders, M.A., and Lens, S.M.A. (2017). Inner centromere localization of the CPC maintains centromere cohesion and allows mitotic checkpoint silencing. Nat Commun 8, 15542.

20. Kawashima, S.A., Yamagishi, Y., Honda, T., Ishiguro, K., and Watanabe, Y. (2010). Phosphorylation of H2A by Bub1 prevents chromosomal instability through localizing shugoshin. Science 327, 172-177.

21. Wang, F., Dai, J., Daum, J.R., Niedzialkowska, E., Banerjee, B., Stukenberg, P.T., Gorbsky, G.J., and Higgins, J.M. (2010). Histone H3 Thr-3 Phosphorylation by Haspin Positions Aurora B at Centromeres in Mitosis. Science.

22. Yamagishi, Y., Honda, T., Tanno, Y., and Watanabe, Y. (2010). Two histone marks establish the inner centromere and chromosome bi-orientation. Science 330, 239-243.

23. Sullivan, B.A., and Karpen, G.H. (2004). Centromeric chromatin exhibits a histone modification pattern that is distinct from both euchromatin and heterochromatin. Nat Struct Mol Biol 11, 1076-1083.

24. Kang, J., Chaudhary, J., Dong, H., Kim, S., Brautigam, C.A., and Yu, H. (2011). Mitotic centromeric targeting of HP1 and its binding to Sgo1 are dispensable for sister-chromatid cohesion in human cells. Mol Biol Cell 22, 1181-1190.

25. Yamagishi, Y., Sakuno, T., Shimura, M., and Watanabe, Y. (2008). Heterochromatin links to centromeric protection by recruiting shugoshin. Nature.

26. Yi, Q., Chen, Q., Liang, C., Yan, H., Zhang, Z., Xiang, X., Zhang, M., Qi, F., Zhou, L., and Wang, F. (2018). HP1 links centromeric heterochromatin to centromere cohesion in mammals. EMBO Rep 19.

27. Eot-Houllier, G., Fulcrand, G., Watanabe, Y., Magnaghi-Jaulin, L., and Jaulin, C. (2008). Histone deacetylase 3 is required for centromeric H3K4 deacetylation and sister chromatid cohesion. Genes Dev 22, 2639-2644.

28. Herz, H.M., Garruss, A., and Shilatifard, A. (2013). SET for life: biochemical activities and biological functions of SET domain-containing proteins. Trends Biochem Sci 38, 621-639. 
29. Kumar, A., Kumari, N., Nallabelli, N., and Prasad, R. (2019). Pathogenic and Therapeutic Role of H3K4 Family of Methylases and Demethylases in Cancers. Indian J Clin Biochem 34, 123132.

30. Han, X., Piao, L., Zhuang, Q., Yuan, X., Liu, Z., and He, X. (2018). The role of histone lysine methyltransferase NSD3 in cancer. Onco Targets Ther 11, 3847-3852.

31. Lucio-Eterovic, A.K., and Carpenter, P.B. (2011). An open and shut case for the role of NSD proteins as oncogenes. Transcription 2, 158-161.

32. Li, Y., Trojer, P., Xu, C.F., Cheung, P., Kuo, A., Drury, W.J., 3rd, Qiao, Q., Neubert, T.A., Xu, R.M., Gozani, O., et al. (2009). The target of the NSD family of histone lysine methyltransferases depends on the nature of the substrate. J Biol Chem 284, 34283-34295.

33. Wagner, E.J., and Carpenter, P.B. (2012). Understanding the language of Lys36 methylation at histone H3. Nat Rev Mol Cell Biol 13, 115-126.

34. Li, W., Tian, W., Yuan, G., Deng, P., Sengupta, D., Cheng, Z., Cao, Y., Ren, J., Qin, Y., Zhou, Y., et al. (2021). Molecular basis of nucleosomal H3K36 methylation by NSD methyltransferases. Nature 590, 498-503.

35. Rahman, S., Sowa, M.E., Ottinger, M., Smith, J.A., Shi, Y., Harper, J.W., and Howley, P.M. (2011). The Brd4 extraterminal domain confers transcription activation independent of pTEFb by recruiting multiple proteins, including NSD3. Mol Cell Biol 31, 2641-2652.

36. Yuan, G., Flores, N.M., Hausmann, S., Lofgren, S.M., Kharchenko, V., Angulo-Ibanez, M., Sengupta, D., Lu, X., Czaban, I., Azhibek, D., et al. (2021). Elevated NSD3 histone methylation activity drives squamous cell lung cancer. Nature 590, 504-508.

37. Kim, S.M., Kee, H.J., Eom, G.H., Choe, N.W., Kim, J.Y., Kim, Y.S., Kim, S.K., Kook, H., Kook, H., and Seo, S.B. (2006). Characterization of a novel WHSC1-associated SET domain protein with H3K4 and H3K27 methyltransferase activity. Biochem Biophys Res Commun 345, 318-323.

38. Angrand, P.O., Apiou, F., Stewart, A.F., Dutrillaux, B., Losson, R., and Chambon, P. (2001). NSD3, a new SET domain-containing gene, maps to $8 p 12$ and is amplified in human breast cancer cell lines. Genomics 74, 79-88.

39. Bennett, R.L., Swaroop, A., Troche, C., and Licht, J.D. (2017). The Role of Nuclear ReceptorBinding SET Domain Family Histone Lysine Methyltransferases in Cancer. Cold Spring Harb Perspect Med 7.

40. Zhou, Z., Thomsen, R., Kahns, S., and Nielsen, A.L. (2010). The NSD3L histone methyltransferase regulates cell cycle and cell invasion in breast cancer cells. Biochem Biophys Res Commun 398, 565-570.

41. Vermeulen, M., Eberl, H.C., Matarese, F., Marks, H., Denissov, S., Butter, F., Lee, K.K., Olsen, J.V., Hyman, A.A., Stunnenberg, H.G., et al. (2010). Quantitative interaction proteomics and genome-wide profiling of epigenetic histone marks and their readers. Cell 142, 967-980.

42. Zentner, G.E., and Henikoff, S. (2013). Regulation of nucleosome dynamics by histone modifications. Nat Struct Mol Biol 20, 259-266.

43. Linares-Saldana, R., Kim, W., Bolar, N.A., Zhang, H., Koch-Bojalad, B.A., Yoon, S., Shah, P.P., Karnay, A., Park, D.S., Luppino, J.M., et al. (2021). BRD4 orchestrates genome folding to promote neural crest differentiation. Nat Genet 53, 1480-1492.

44. Shen, C., Ipsaro, J.J., Shi, J., Milazzo, J.P., Wang, E., Roe, J.S., Suzuki, Y., Pappin, D.J., JoshuaTor, L., and Vakoc, C.R. (2015). NSD3-Short Is an Adaptor Protein that Couples BRD4 to the CHD8 Chromatin Remodeler. Mol Cell 60, 847-859.

45. Lopez-Serra, L., Kelly, G., Patel, H., Stewart, A., and Uhlmann, F. (2014). The Scc2-Scc4 complex acts in sister chromatid cohesion and transcriptional regulation by maintaining nucleosome-free regions. Nat Genet 46, 1147-1151.

46. Muñoz, S., Minamino, M., Casas-Delucchi, C.S., Patel, H., and Uhlmann, F. (2019). A Role for Chromatin Remodeling in Cohesin Loading onto Chromosomes. Mol Cell 74, 664-673 e665.

47. Muñoz, S., Passarelli, F., and UhImann, F. (2020). Conserved roles of chromatin remodellers in cohesin loading onto chromatin. Curr Genet 66, 951-956. 
48. Manning, B.J., and Yusufzai, T. (2017). The ATP-dependent chromatin remodeling enzymes CHD6, CHD7, and CHD8 exhibit distinct nucleosome binding and remodeling activities. J Biol Chem 292, 11927-11936.

49. Hakimi, M.A., Bochar, D.A., Schmiesing, J.A., Dong, Y., Barak, O.G., Speicher, D.W., Yokomori, K., and Shiekhattar, R. (2002). A chromatin remodelling complex that loads cohesin onto human chromosomes. Nature 418, 994-998.

50. Kagey, M.H., Newman, J.J., Bilodeau, S., Zhan, Y., Orlando, D.A., van Berkum, N.L., Ebmeier, C.C., Goossens, J., Rahl, P.B., Levine, S.S., et al. (2010). Mediator and cohesin connect gene expression and chromatin architecture. Nature 467, 430-435.

51. Zuin, J., Franke, V., van Ijcken, W.F., van der Sloot, A., Krantz, I.D., van der Reijden, M.I., Nakato, R., Lenhard, B., and Wendt, K.S. (2014). A cohesin-independent role for NIPBL at promoters provides insights in CdLS. PLoS Genet 10, e1004153.

52. Kuo, A.J., Cheung, P., Chen, K., Zee, B.M., Kioi, M., Lauring, J., Xi, Y., Park, B.H., Shi, X., Garcia, B.A., et al. (2011). NSD2 links dimethylation of histone $H 3$ at lysine 36 to oncogenic programming. Mol Cell 44, 609-620.

53. Zhu, L., Li, Q., Wong, S.H., Huang, M., Klein, B.J., Shen, J., Ikenouye, L., Onishi, M., Schneidawind, D., Buechele, C., et al. (2016). ASH1L Links Histone H3 Lysine 36 Dimethylation to MLL Leukemia. Cancer Discov 6, 770-783.

54. Sumara, I., Vorlaufer, E., Gieffers, C., Peters, B.H., and Peters, J.M. (2000). Characterization of vertebrate cohesin complexes and their regulation in prophase. J Cell Biol 151, 749-762.

55. Watrin, E., and Legagneux, V. (2005). Contribution of hCAP-D2, a non-SMC subunit of condensin I, to chromosome and chromosomal protein dynamics during mitosis. Mol Cell Biol 25, 740-750.

56. Kitajima, T.S., Hauf, S., Ohsugi, M., Yamamoto, T., and Watanabe, Y. (2005). Human Bub1 defines the persistent cohesion site along the mitotic chromosome by affecting Shugoshin localization. Curr Biol 15, 353-359.

57. Schmitz, J., Watrin, E., Lenart, P., Mechtler, K., and Peters, J.M. (2007). Sororin Is Required for Stable Binding of Cohesin to Chromatin and for Sister Chromatid Cohesion in Interphase. Curr Biol.

\section{Figure legends}

Figure 1: NSD3 prevents premature sister chromatids separation in mitosis. (A) Representative images of chromosome spreads with magnified chromosome from HeLa cells transfected for $72 \mathrm{~h}$ with control or different SET domain methyltransferase siRNA. (B) Western blotting analysis of NSD3 depletion after 72h siRNA transfection. RAD2 1 and $\alpha$-Tubulin were used as nuclear and cytoplasmic loading marker, respectively. Table indicates targeted exons by respective siRNA. Note the presence of a non-specific band just below the short NSD3s isoform band. (C) For each siRNA tested, cells displaying sister chromatid separation were scored. 300-500 prometaphase cells were counted in each experiment and the mean and SD of relative amount of prometaphase cells with cohesion defects for 5 experiments is represented.

Figure 2: NSD3 contribution to cohesion maintenance occurs before mitotic entry. (A-B) Immunofluorescence with BUB1 (A) and SGO1 (B) antibodies after 72h NSD3 siRNA treatment. CREST serum were used to label centromere position. Scale bars correspond to 10 $\mu \mathrm{m}$. (C-E) Measurement of inter-centromeres distance on chromosome 11 following NSD3 and 
RAD 21 depletion. After 8h siRNA transfection, cells have been subjected to a double thymidine-block, followed by a 6 h release to enrich in $\mathrm{G} 2$ cells. Then, whole-cell extracts were prepared for western blot to check for protein extinction (C) or FISH were performed on cells with a specific probe for centromeric chromosome 11 alpha-satellite sequence (D-E). (D) Representative images of FISH experiments with $4 \mathrm{X}$ magnified view of one the three chromosome 11 centromere loci. (E) Box plot representation of the distance between paired FISH signals. ( $\mathrm{n}=162$ for each tested siRNA from three independent experiments, 5-95 percentiles are indicated).

Figure 3: NSD3 depletion reduces cohesin and kollerin loading at mitotic exit. Synchronized control or NSD3-depleted cells were arrested in mitosis by nocodazole treatment. Mitotic cells were collected by shake-off and released from the arrest. At the indicated time points, chromatin-bound fractions were prepared and analyzed by western-blot. Topoisomerase II was used as a control for chromatin-associated proteins. p-H3S10 and CAP-D2 were analyzed to monitor progression through mitosis. Histones were stained with Coomassie to show the equivalence of protein amount between lanes. Representative capture of western-blot are shown on the left part of the panel. Quantification of signal intensity of the corresponding images are represented on the right part of the panel in black or red for cells treated with Luc or NSD3 siRNA, respectively.

Figure 4: NSD3 localization during the cell cycle. Proliferative HeLa cells were fixed immediately (A) or after a 1 min $0.1 \%$ triton incubation to extract the cell soluble content (B). (C) Representative images of interphasic or mitotic cells transfected for 72h with Luc or NSD3 siRNA. Cells were swollen by hypotonic treatment and spotted on a slide by cyto-centrifugation before immuno-labelling with NSD3 and CREST serum. (D) and (E): Representative images from metaphase to telophase of LAP-NSD3L expressing cells immuno-labelled with RAD21 (D) or MAU2 (E). For all image panels, scale bars correspond to $10 \mu \mathrm{m}$.

Figure 5: Depletion of NSD3 long isoform induces premature separation of sister chromatids. (A) Western-blot analysis of specific NSD3 isoforms depletion efficiency after $72 \mathrm{~h}$ transfection with the indicated siRNA. RAD21 and actin were used as nuclear and cellular loading marker, respectively. Note that, at the long exposure time used to ensure that NSD3s is properly depleted, the NSD3s band and the non-specific band observed just below appear to be fused in Luc, NSD3L-A and NSD3L-B lanes. (B) Percentage of prometaphase with separated sister chromatids in cells depleted for specific NSD3 isoforms. Data represents mean and SD of three independent experiments. 150-300 prometaphase cells were counted for each condition in individual experiments. (C) Representative images of the nuclear presence of inducible LAPNSD3 after doxycyclin induction in the LAP-NSD3L HeLa cell line. Scale bar correspond to 
692

$20 \mu \mathrm{m}$. (D) Western-blot analysis of the various exogenous and endogenous form of NSD3 following endogenous NSD3 depletion and concomitant LAP-NSD3L induction in the LAPNSD3L HeLa cell line. LAP-tag fused with NSD3L generates a heavier band than NSD3L which is detected only following doxycyclin induction. $\alpha$-Tubulin and MAU2 were used as cytoplasmic and nuclear loading marker, respectively. (E) Representative images of a microscope field with cells expressing (1) or not (2) LAP-NSD3L after 72h doxycyclin induction and immuno-labelling with anti-GFP antibodies to improve signal detection. The left panel shows GFP labelling merged with CREST serum and DNA signals. 9X magnification of a representative paired centromeric region were shown for each indicated mitotic cell. On the right panel, the percentage of prometaphases displaying separated sister chromatids is shown following $72 \mathrm{~h}$ siRNA depletion. Data is displayed according to doxycyclin induction and the presence or the absence of GFP labeling in the doxycyclin induced LAP-NSD3L HeLa cells. Data represents mean and SD of three independent experiments. 100-300 prometaphase cells were counted for each condition in individual experiments.

\section{Supplemental figure legends}

Figure S1: Screening for contribution of SET domain methyltransferases to prevent sister chromatid separation in mitosis. For each of the 14 evaluated methyltransferases, 3 different siRNA were tested. Cells were transfected for $72 \mathrm{~h}$ before fixation and chromosome spreading. 300-500 prometaphase cells were counted and the proportion of mitotic cells with separated chromatids was assessed according to the phenotypes shown in figure $1 \mathrm{~A}$.

\section{Figure S2: NSD3 depletion does not affect cohesin and MAU2 expression level or global} di-methylation level of H3K4 and H3K36. Synchronized control or NSD3-depleted cells were arrested in mitosis by nocodazole treatment as indicated in the figure. Mitotic cells were then collected by shake-off and released from the arrest. At the indicated time points, whole-cell extracts were prepared and analyzed by western-blot. $\alpha$-Tubulin and Histone H4 were used as cytoplasmic and nuclear loading marker, respectively. Decrease in p-H3S10 signal indicated the efficiency of nocodazole release.

Figure S3: Efficiency of NSD3 depletion shown by immunofluorescence. HeLa cells were transfected with the indicated siRNA for $48 \mathrm{~h}$ and soluble cell content was pre-extracted before immunofluorescence. Scale bar corresponds to $10 \mu \mathrm{m}$.

Figure S4: Cellular localization of LAP-NSD3L during the cell cycle. Proliferative LAPNSD3L HeLa cell line was fixed immediately (left panel) or after a 1 min $0.1 \%$ triton incubation to extract cell soluble content (right panel). Cells were immuno-labeled for p-H3S10 to identify 
730 precisely the different mitotic phases. Representative images are shown and scale bar 731 correspond to $10 \mu \mathrm{m}$.

Figure S5: Timing of LAP-NSD3L, MAU2 and RAD21 recruitment onto chromatin telophase of LAP-NSD3L expressing cells that were pre-extracted before immunolabelling with RAD21 (A) or MAU2 (B) as indicated in the material and methods section a. For all image panels, scale bars correspond to $10 \mu \mathrm{m}$. 


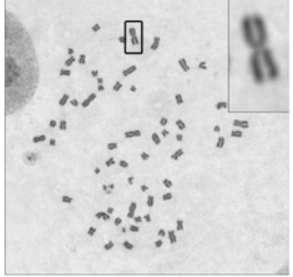

cohesion defects
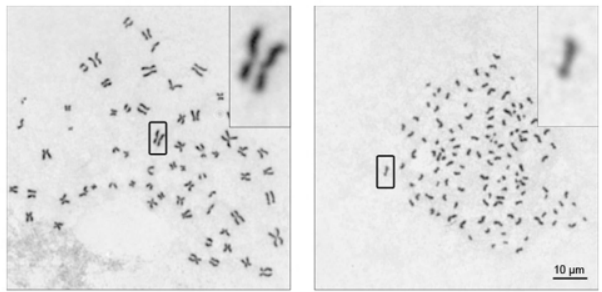

$10 \mu \mathrm{m}$

\begin{tabular}{|l|c|c|c|}
\hline siRNA & siRNA NSD3- $\alpha$ & siRNA NSD3- $\beta$ & siRNA NSD3- $\gamma$ \\
\hline Targeted exon & 5 & 6 & 2 \\
\hline
\end{tabular}
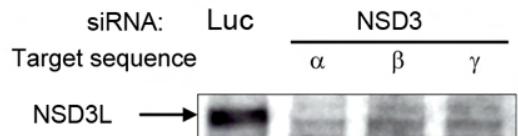

NSD3s

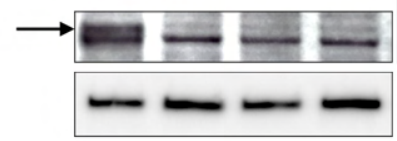

WB: NSD3

WB: RAD21

WB: $\alpha$-Tubulin

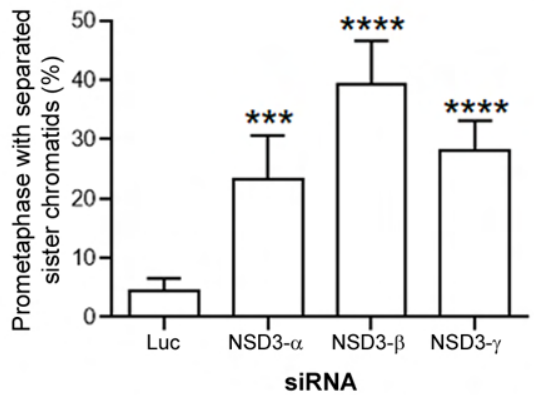

Figure 1: NSD3 prevents premature sister chromatids separation in mitosis 
A

SiRNA

Luc

SiRNA

NSD3

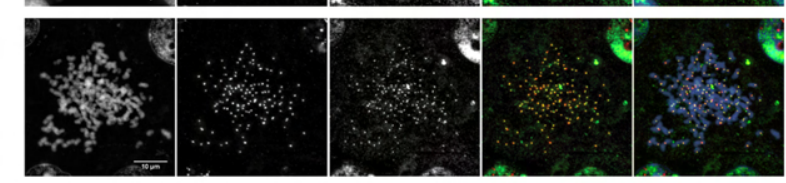

D

siRNA Thymidine ReleaseThymidine transfection Block

$\longleftarrow$ NSD3 L

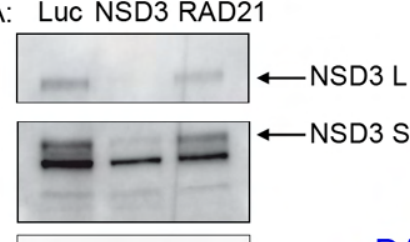

WB: RAD21

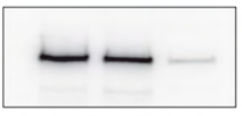

WB: $\beta$-Tubulin

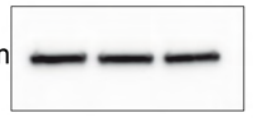

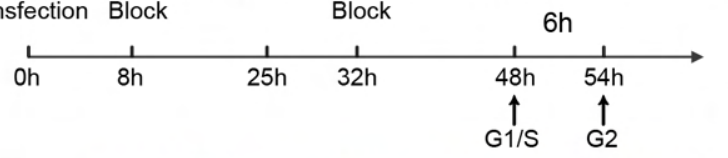

SiRNA Luc

SiRNA NSD3

siRNA RAD21

DAPI

Cen-Chr11
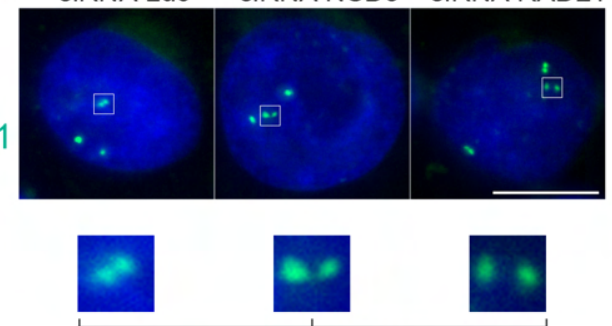

E

Release FISH

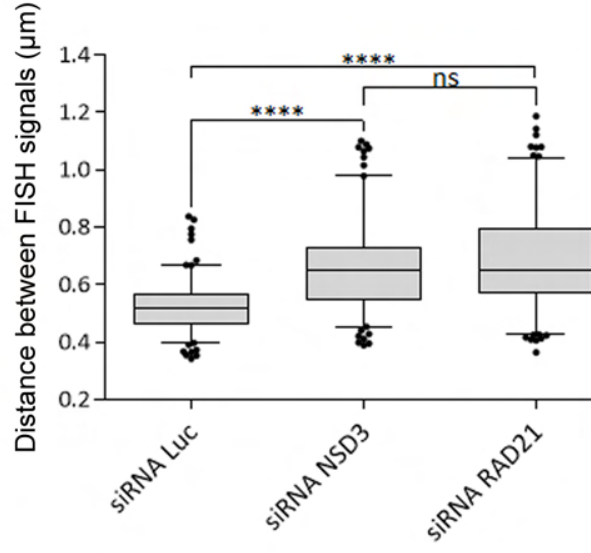



transfection Block Nocodazole

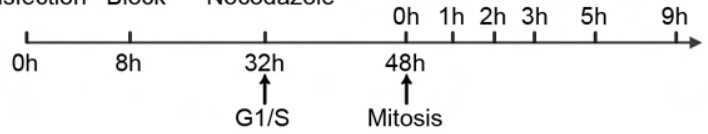

B

Time after nocodazole release $(h)$

NSD3L

NSD3s

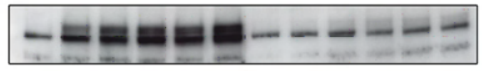

MAU2

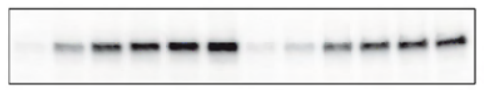

RAD21

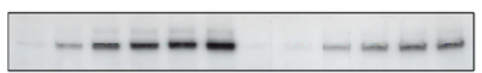

SA2

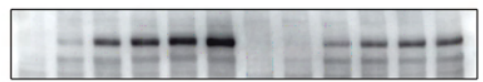

SMC3

Topoisomerase 2

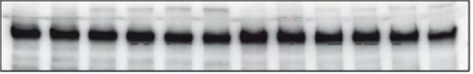

CAPD2 (condensin)
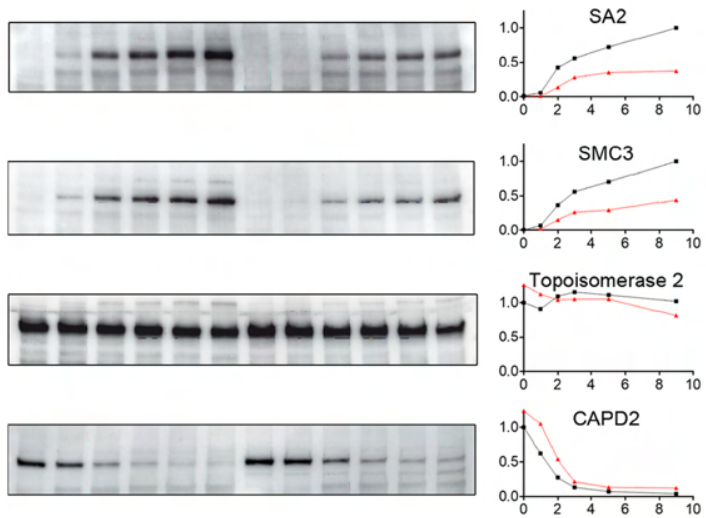

p-H3S10
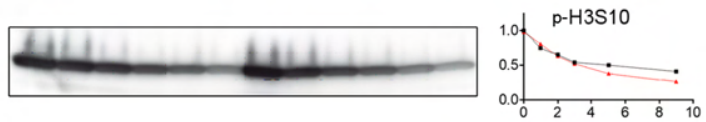

Histones

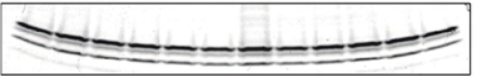

Time after nocodazole release $(\mathrm{h})$

Figure 3: NSD3 depletion reduces cohesin and kollerin loading at mitotic exit 
$D$

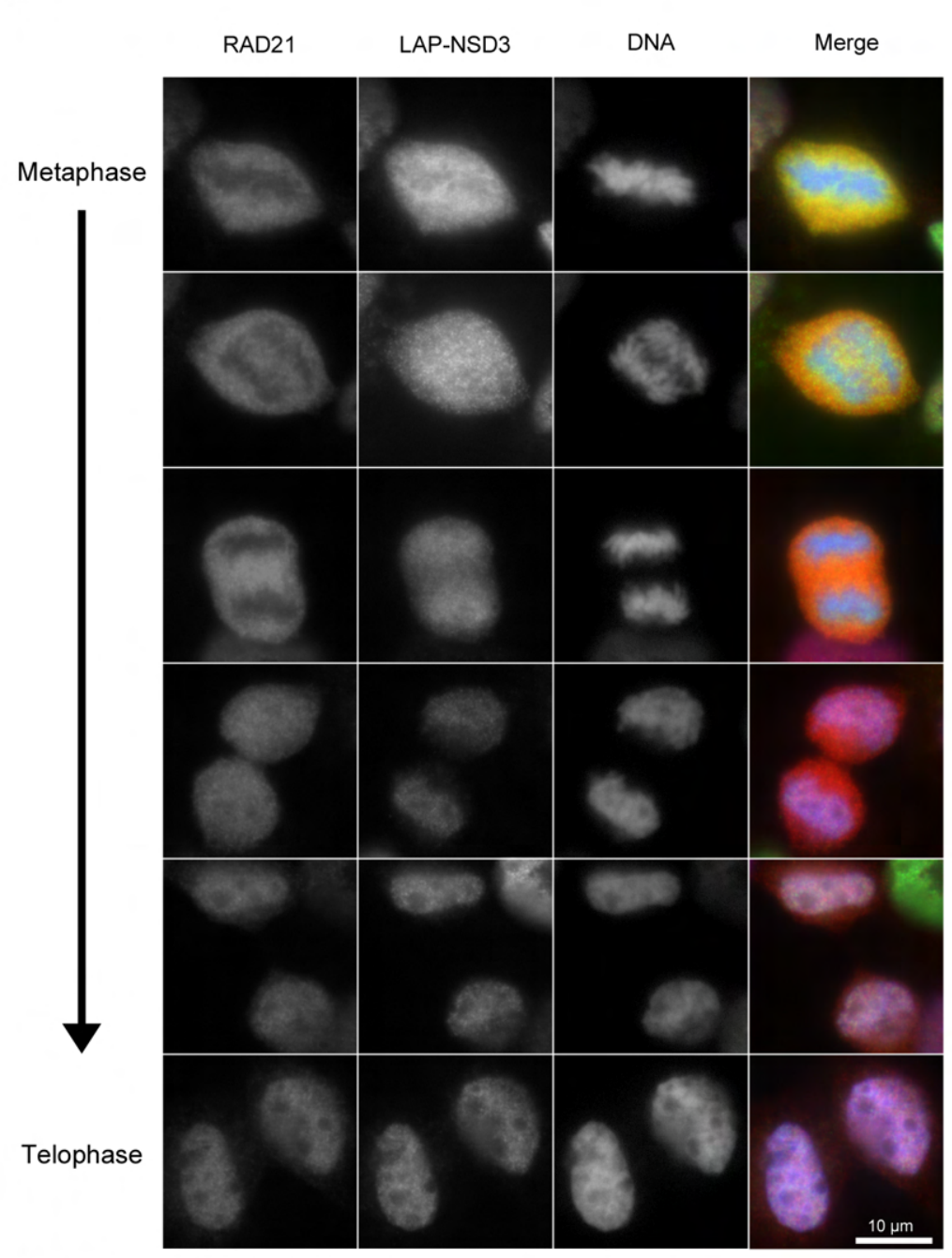

E

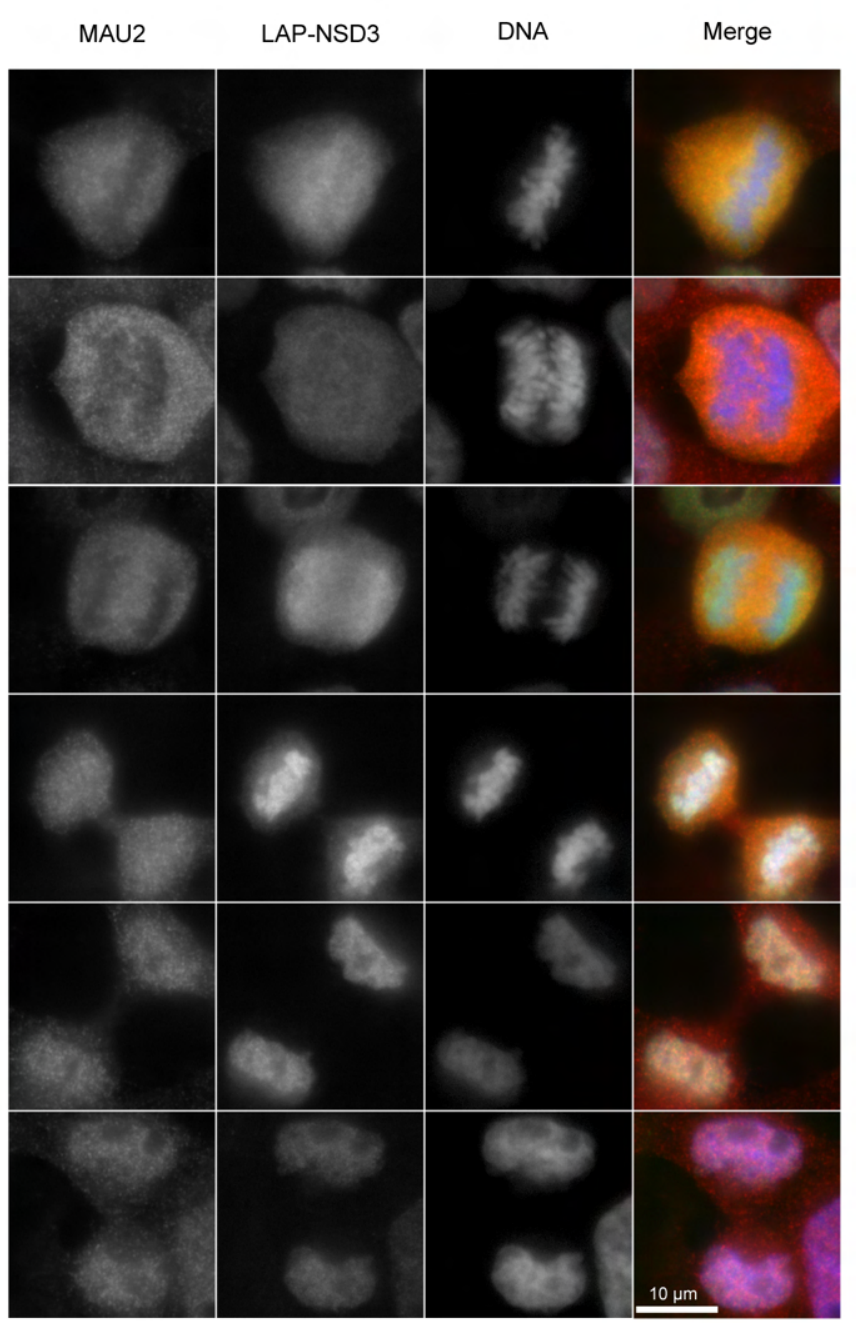

Figure 4 extended: NSD3 localization during the cell cycle 
A

get sequence: Luc Both NSD3 forms NSD3L NSD3s

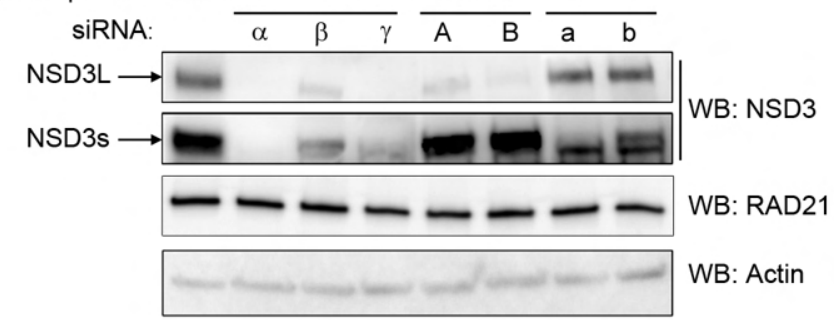

LAP-NSD3L

Merged with DNA

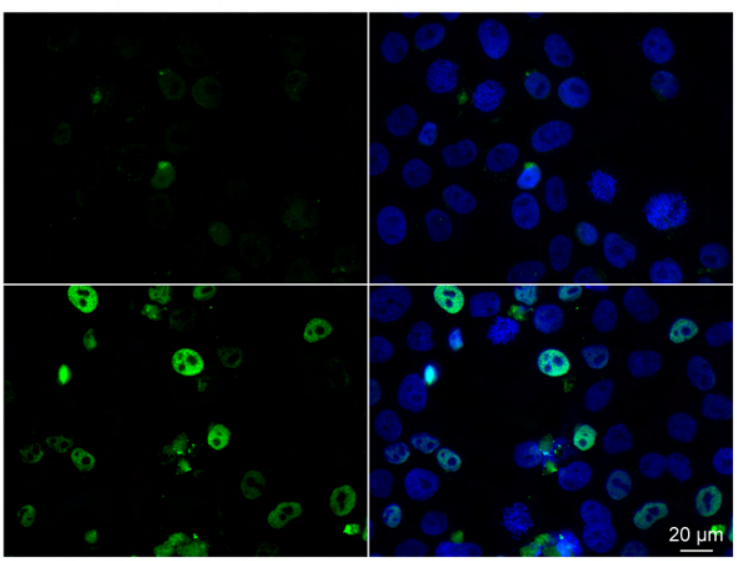

E
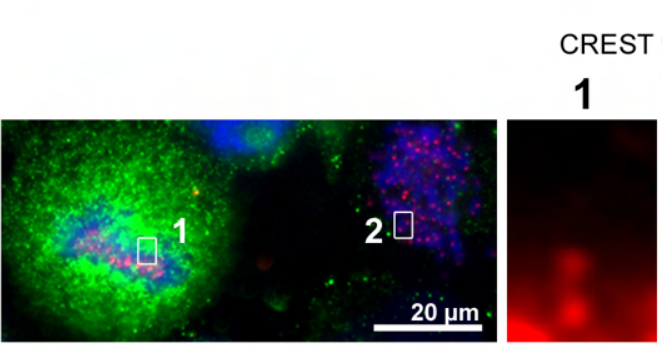

DNA CREST LAP-NSD3L

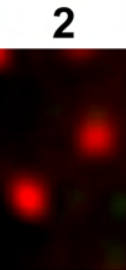

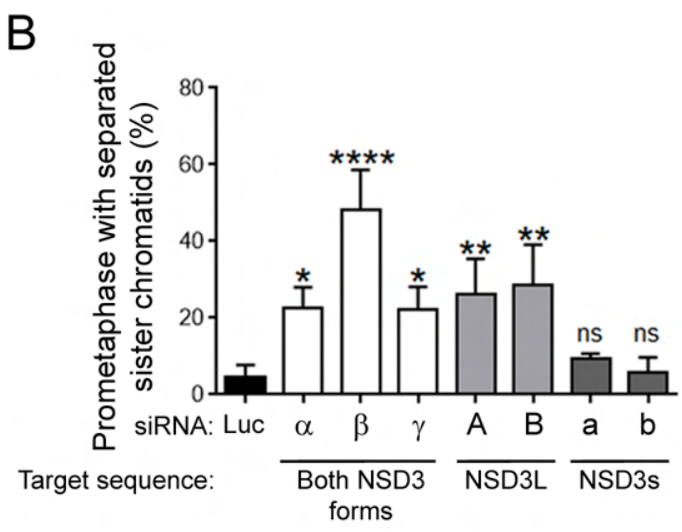

D

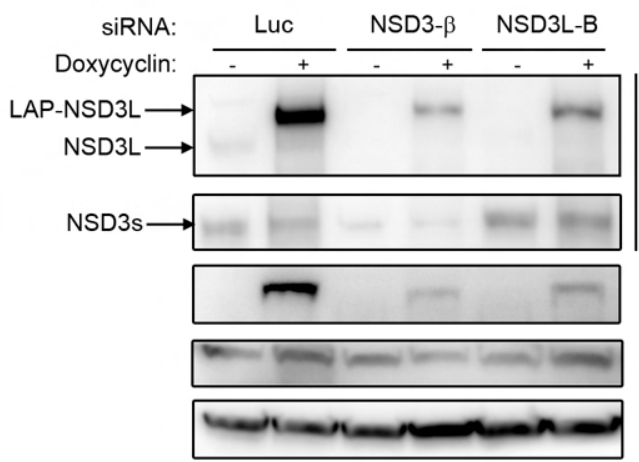

WB: NSD3

WB: GFP

WB: MAU2

WB: $\alpha$-Tubulin

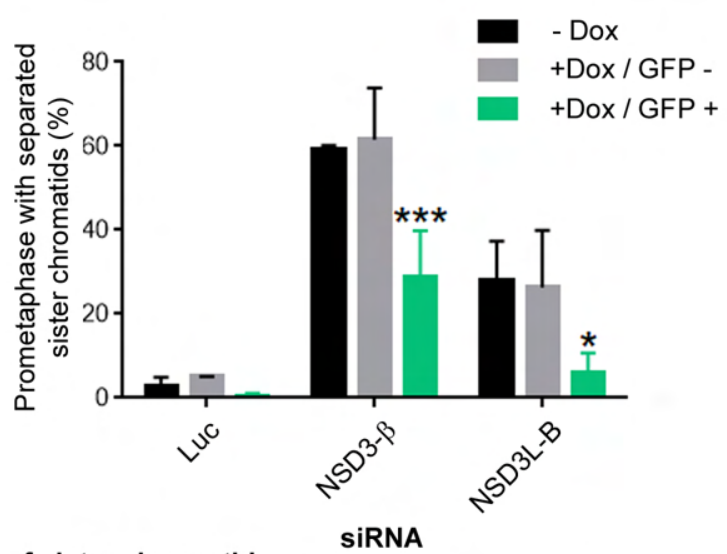

Figure 5: Depletion of NSD3 long isoform induces premature separation of sister chromatids 


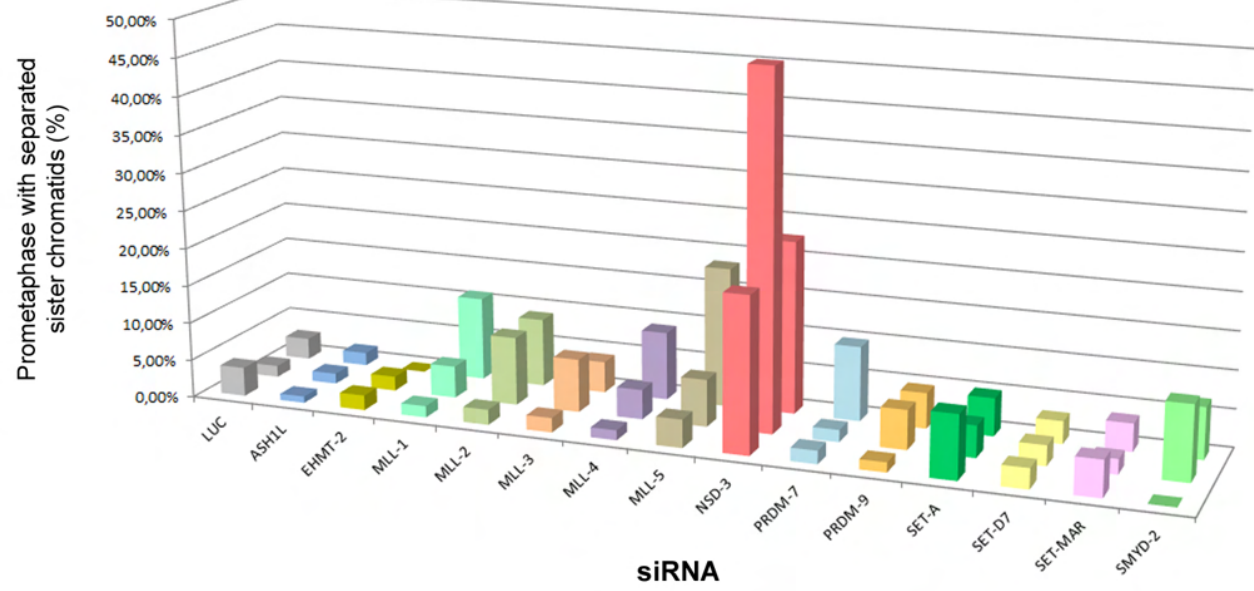

Figure S1: Screening for contribution of SET domain methyltransferases to prevent sister chromatid separation in mitosis 
siRNA Thymidine Release + Release transfection Block Nocodazole

\section{Extraction time}

Oh $\quad$ 8h $\quad$ in

Oh $1 \mathrm{~h} 2 \mathrm{~h} 3 \mathrm{~h} \quad 5 \mathrm{~h}$

G1/S

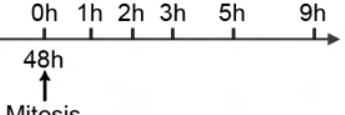

Whole cell extracts

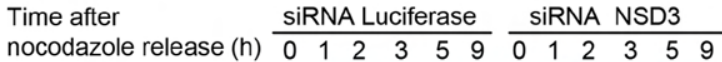

NSD3L

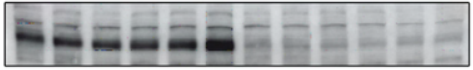

NSD3s

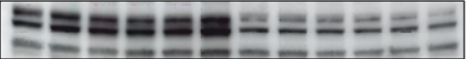

RAD21 ---------1

SA2

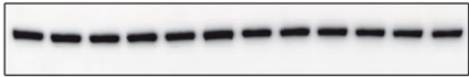

SMC3

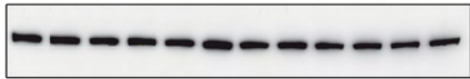

MAU2

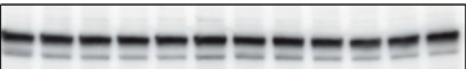

$\alpha$-Tubulin

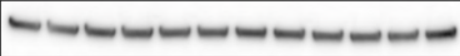

$\mathrm{p}-\mathrm{H} 3 \mathrm{~S} 10$

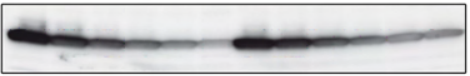

H3K36me2

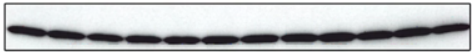

$\mathrm{H} 3 \mathrm{~K} 4 \mathrm{me} 2$

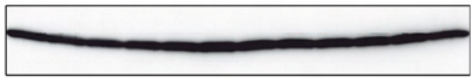

$\mathrm{H} 4$

Figure S2: NSD3 depletion does not affect cohesin and MAU2 expression level or global di-methylation level of H3K4 and H3K36 
$A$

\section{pre-extraction}

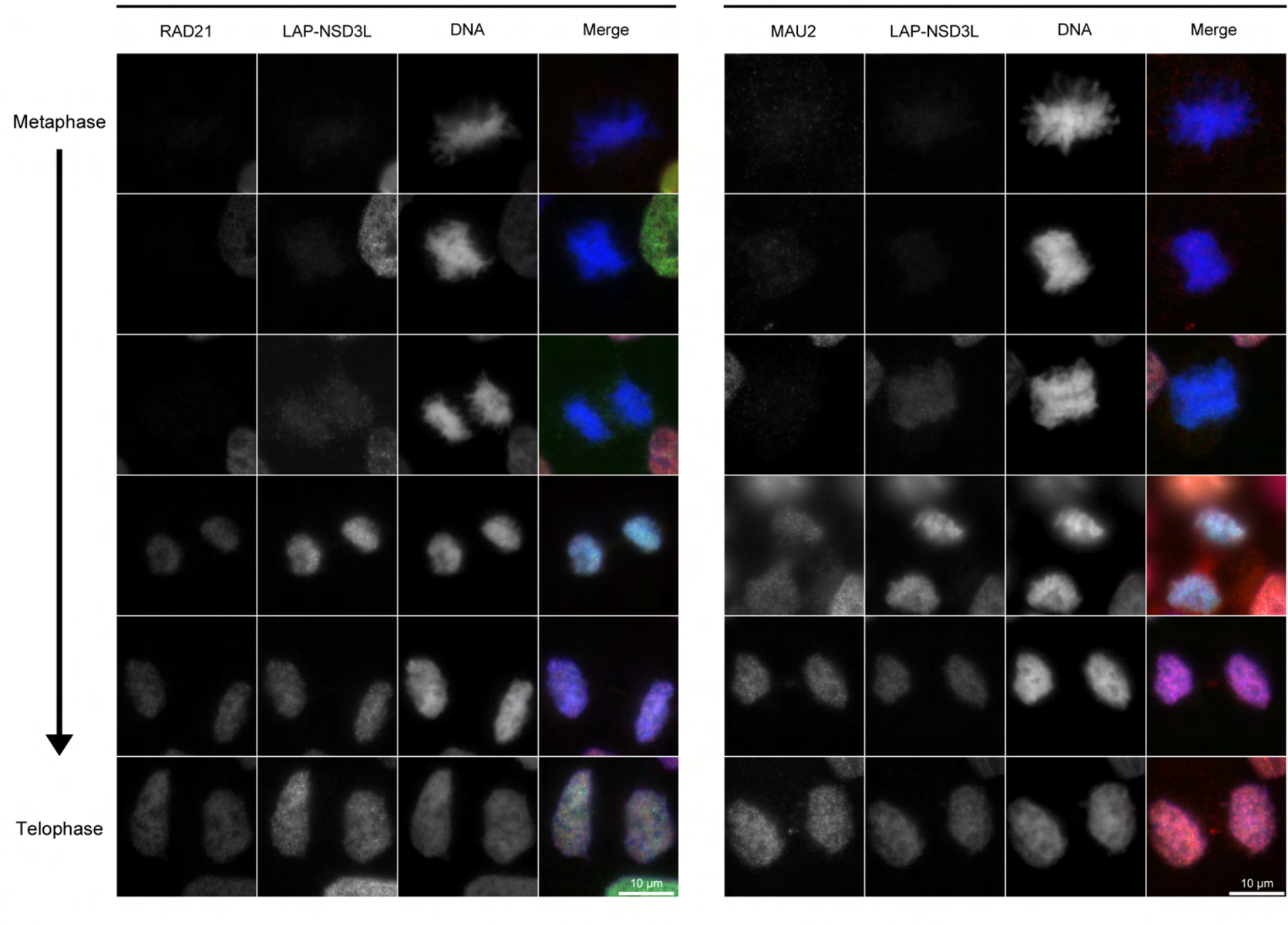

B

pre-extraction

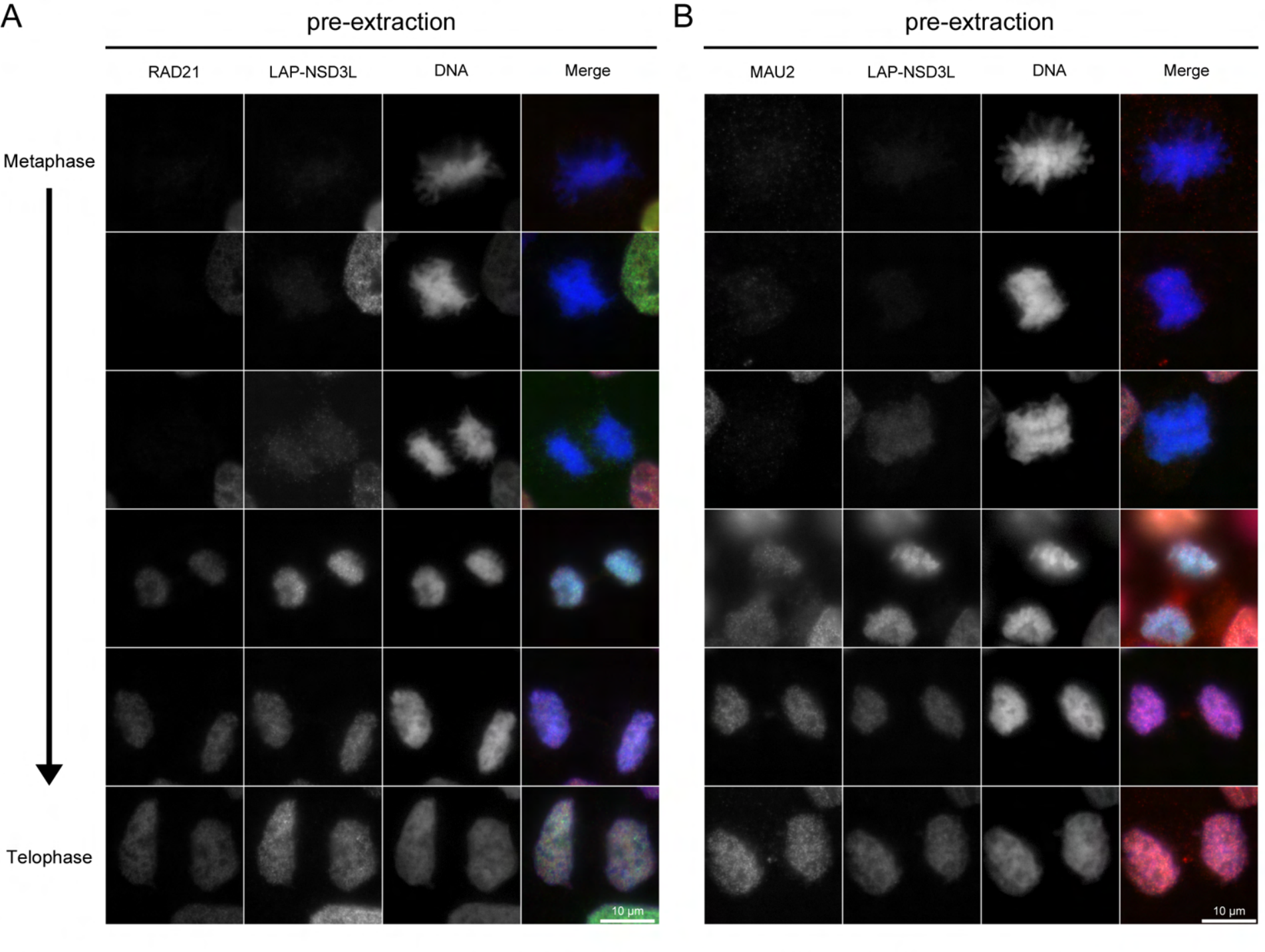

Figure S5: Timing of LAP-NSD3L, MAU2 and RAD21 recruitment onto chromatin following metaphase-anaphase transition

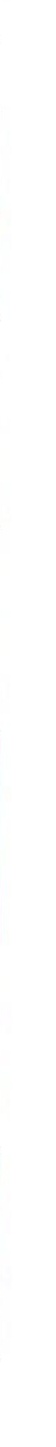

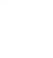

